\title{
Sedimentomics - exploring the microbial treasures in deep marine environments
}

\begin{abstract}
Advancements in metagenomics and related molecular approaches has allowed easy access to extreme and less explored vicinities over the globe, one of the best examples being the deep marine sediment microbial communities. Several recent investigations have provided access to high number of genomic and metagenomic datasets from circumnavigation in different water bodies that form a major portion of the Earth's surface area. And these microbes play a significant role in the biogeochemical cycles, biodegradation of hydrocarbons and most importantly help in elucidating the vast arena of antibiotics and antibiotic resistance that poses immense health risk to humans. Here, we summarize the advancements in deep sediment microbiome studies using the '-omic' technologies and the remarkable functional and community observations that have enriched the marine microbes' database in an extremely short span of time.
\end{abstract}

Keywords: deep sediment, marine, microbiome, omics, genome reconstruction, bio prospection
Volume 3 Issue 2 - 2016

\author{
Neelam M Nathani,' Chandra Shekar \\ Mootapally, ${ }^{2}$ Bharti P Dave' \\ 'Department of Life Sciences, Maharaja Krishnakumarsinhji \\ Bhavnagar University, India \\ ${ }^{2}$ Department of Marine Science, Maharaja Krishnakumarsinhji \\ Bhavnagar University, India \\ \#Authors have contributed equally
}

\begin{abstract}
Correspondence: Neelam M Nathani, Department of Life Sciences, Maharaja Krishnakumarsinhji Bhavnagar University, Bhavnagar, Gujarat, India, Email neelam.nathani@yahoo.com Bharti P Dave, Department of Life Sciences, Maharaja Krishnakumarsinhji Bhavnagar University, Bhavnagar, Gujarat, India, Email bpd8256@yahoo.com
\end{abstract}

Received: September 29, 2016 | Published: November 03, 2016

\section{Introduction}

Our planet is majorly dominated by the marine environments. The marine microbial diversity holds extreme importance with several biotechnological applications including the proper driving of the biogeochemical cycles. However, the marine microbes are less explored as $>90 \%$ are uncultivable in the laboratory conditions, making it inevitable to rely on the advanced 'omic' technologies to uncover the dearth of information on the genetic diversity of the marine microbes. ${ }^{s}$ Metagenomics, that includes direct nucleic acid study of the total environment, has led to identification of novel microbes ${ }^{2,3}$ potential metabolic pathways, ${ }^{4}$ and several highly expressing enzymes ${ }^{5}$ for varied applications apart from the general information about the microbial community and functional structure of the respective niches. ${ }^{6}$ Thus, metagenomics and genomics based research studies are increasing for exploration of the marine microbes. The former statement is well proved based on the numerical data (as on 1st September, 2016) on the research publications observed using the search terms "deep sea microbes" and "deep sediment microbes" in Pubmed Central archive, which revealed that the publications in these areas have reached $>80 \%$ in the last five years (Number of publications 3004 and 1730, respectively) as compared to the total publications in the previous decade (Number of publications 3784 and 2102, respectively), with most of the research outcomes based on the metagenomics and genomics based approaches. Thus, these advances are unraveling the distinguished features of these deep marine microbiome to that of the other niches.

Deep sediment microbes: Abundance, unique adaptability and functional diversity

Bacteria and archaea are among the most abundant microbes of the marine environments with thousands of different species observed in a single sample. The variabilities affecting the bacterial composition is very high in marine environments leading to distinct communities based on the location, depth, salinity, temperature, nutrient/energy availability, sedimentation, etc. number of factors as compared to other niches. ${ }^{7}$ The diversity in the sediments is observed to be more compared to that in the deep waters, and specifically, those in the oceanic dessert areas is found to be the least due to very less nutrient circulation and subsequent lower primary productivity. ${ }^{8}$ Also, the marine benthic and pelagic communities are observed to be significantly diverse in the bacterial species abundance. The marine metagenomic endeavors have revealed that the Proteobacter is the pre-dominant phyla in the deep marine biosphere with Alphaproteobacter like the Rhodobacter clad (RCB) like microbes specifically dominating the deeper marine environment and exhibiting novel degradation pathways. ${ }^{9}$ Also, apart from the bacteria, marine fungi, specifically in the deep, has grabbed the attention of few of the research groups, as the -omic technologies has allowed identification of novel fungus using the ITS (Internal transcribed spacer) sequences as marker for fungal identification and classification. Ascomycota and Basidiomycota have been observed to be the dominant fungi. ${ }^{10}$ An interesting observation based on these molecular studies is that these marine fungi are observed to be highly active in the carbon cycle, and also the observed classes in the marine deep biosphere are common to the terrestrial fungi except the morphological adaptations to survive in the water. All these are stating a very good example of extreme advancements in the molecular techniques and relevant improvements in the knowledge of the marine microflora which can be termed as the 'Sedimentomics'.

A related branch of work, includes the assessment of the microbial structure in contaminated and/or polluted marine environments. The water bodies are highly susceptible to pollution and contamination due to numerous pyrogenic and petrogenic sources. The increase in anthropogenic activities is constantly causing the disturbance in the physicochemical parameters of the water and sediments leading to reduced quality for microflora persistence. Thus, the microbial 
community structure and dynamics at these areas and those away from such disturbance is likely to be varied. The contaminants are usually hydrophobic and dense in nature and thus are bound to deposit in the sediments thus posing a profound effect on the sediment microbes. Metagenomic analysis of the deep sediments $(\sim 1500 \mathrm{~m})$ of the Deepwater Horizon oil spill (2010), Gulf of Mexico showed that the microbial metabolic potentials to degrade hydrocarbons were highly enhanced, indicative of the ability of indigenous sediment bacteria to degrade them, with specific bacterial communities viz., uncultured Oceanospirillales found to be dominating post spillage revealing their role in the biodegradation of such contaminated sediments. ${ }^{11}$ It is clear from the details mentioned above, that metagenomics is by leaps and bounds increasing the knowledge of novel marine microbes, their unique metabolic potentials for several applications to improve the marine ecosystem and for human benefits. However, one of the major significance of the sedimentomics study is the exploration of wide range of antibiotics and throwing light on the antibiotic resistant gene (ARG) profile of quality stressed water niches. Recent studies have thrown light on the distinct microflora profile and the antimicrobial resistance pattern in sediments. One such study from the Oluwa river, which is the major water source to the residents, revealed the possibility of horizontal gene transfer of such ARGs due to anthropogenic activities. ${ }^{12}$ One mores study on fish farm sediments observed putative ARGs in the sediment plasmids. With an important observation that few of the sequences were quite identical with transposons suggesting acquisition from human pathogens. ${ }^{13}$

Apart from the benefits there are several caveats to be considered for these kind of study expeditions and also the awareness about the increasing need of computational facilities to handle this huge sequence data. One of the major cautious step for deep marine sediment microbiota study includes the sampling methodology. As each environment has its own properties based on the water body type, be it estuarine, or river. Based on the sedimentation process the type of sediment would also vary. Another variable includes the study objective as each layer of sediment form surface sediment up to few centimeters has a varied microbial diversity. In context to the same, there are multicorers, grab samplers, and box corers available for efficient sampling of sediment for -omic studies. Once the sampling is performed, next the NGS platform to be chosen is a vital point of consideration. And lastly, sequencing produces enormous data for analysis and interpretation, which is the most challenging task requiring a team effort to draw conclusive interpretation to understand the microbe-environment relationship. The bioinformaticians are on the toes with almost an updated algorithm/softwares visible every week for the analysis and better interpretation of metagenomic and genomic outputs. CAMERA, the metagenomic resource for microbial ecology currently hosts the data of about 147 marine metagenome and genome data from different environments such as the open oceans, estuaries, bays, and marine host associated microbiota. The databases are now being poured several marine related gene and RNA sequences daily due to the availability of advanced softwares.

\section{Marine genomes assembled from metagenomic datasets}

Genome assembly from metagenomic data is a step ahead to identify the Candidatus phyla, which were hitherto unknown on account of their unculturability in vitro. The genomes of individual species are assembled by either the sequence dependent or independent approaches based on the microdiversity levels of the niche under study. Several parameters like GC, k-mer frequencies, single copy genes and scaffold coverage are used to bin the sequences of unique phylum.
This has led to a great increase the marine bacterial genomes adding several new groups with versatile capabilities in the evolutionary tree of life. By this approach, 290 genomes of Candidatus marine bacteria have been reconstructed from the Tara oceansmetagenomes that have covered 210 different ecosystems. ${ }^{14}$ Among the 290 genomes, 14 are novel Prochlorococcus and Synechococcus genus of Cyanobacteria and 33 novel SAR11 genomes, which has been a dominating clad observed in numerous deep marine studies. ${ }^{15}$ And other striking outcome included 40 clustered bins that lacked the single copy genes suggesting their being viral sequences which were termed as 'giant viruses' by the team of scientists. Similarly, nine genomes have also been assembled from the South Pacific Gyre, the most oligotrophic location among the marine environments that revealed that these genomes were potentially active to transform various organic matter in the deep location.

\section{Conclusion}

This mini review summarizes in brief the current raise in the microbial ecology of the deep sediments in combination with the advanced-omic technologies. It highlights the increase in the database and research publications of this particular environments, which were before few years considered as highly distant and difficult to explore regions of the globe. It highlights the diversity of the marine microbes due to several variable factors and the potentiality of novel marine bacteria in degrading hydrocarbons as a part of bioremediation of pollutant areas and in maintaining the biogeochemical cycle of elements in the marine ecosystem. It also states the importance and need of several interdisciplinary research projects to discover the role of sediment bacteria in the comprehensive antibiotic resistance profile, especially the deep sediment microbes.

\section{Acknowledgments}

This research area was supported by the Science and Engineering Research Board, Government of India, in the form of Early Career Research Award-National Post Doctoral Fellowship to NMN (Grant No. PDF/2016/000190) and CSM (Grant No. PDF/2016/001239).

\section{Conflict of interest}

Author declares that there is no conflict of interest.

\section{References}

1. Roesh LFW, Fulthorpe RR, Riva A, et al. Pyrosequencing enumerates and contrasts soil microbial diversity. ISME J. 2007;4:739-751.

2. Trindade M, Van Zyl L, Navarro Fernandes J, et al. Targeted metagenomics as a tool to tap into marine natural product diversity for the discovery and production of drug candidates. Front Microbiol. 2015;6:890.

3. Nathani NM, Duggirala SM, Chandra Shekar M, et al. Isolation of chitinolytic Clostridium sp NCR from Mehsani buffalo rumen, its genomic analysis and potential role in rumen. Genom Data. 2015;5:109-111.

4. Nathani NM, Duggirala SM, Bhatt VD, et al. Correlation between genomic analyses with metatranscriptomic study reveals various functional pathways of Clostridium sartagoforme AAU1, a buffalo rumen isolate. J Applied Animal Research. 2016;44(1):498-507.

5. Mootapally CS, Nathani NM, Patel AK, et al. Mining of ruminant microbial phytase (RPHY1) from metagenomic data of mehsani buffalo breed: identification, gene cloning, and characterization. $J \mathrm{Mol}$ Microbiol Biotechnol. 2016;26(4):252-260. 
6. Nathani NM, Patel AK, Chandra Shekar M, et al. Effect of roughage on rumen microbiota composition in the efficient feed converter and sturdy Indian Jaffrabadi buffalo (Bubalusbubalis). BMC Genomics. 2015;16:1116

7. Rusch DB, Halpern AL, Sutton G, et al. The sorceree II global ocean sampling expedition: Northwest Atlantic through eastern tropical Pacific. PlosBiol. 2007;5(3):e77.

8. Weng Y, Sheng HF, He Y, et al. Comparison of the levels of bacterial diversity in freshwater, intertidal wetland, and marine sediments by using millions of Illumina tags. Appl Environ Microbiol. 2012;78(23):8264-8271.

9. Parkes RJ, Cragg B, Rousell E, et al. A review of prokaryotic populations and processes in sub-seafloor sediments, including biosphere: geosphere interactions. Marine Geol. 2014;352:409-425.

10. Orsi WD, Biddle JF, Edgcomb V. Deep sequencing of subsea floor eukaryotic rRNA reveals active fungi across marine sub surface provinces. PLoS ONE. 2013;8(2):e56335.
11. Mason OU, Scott NM, Gonzalez A, et al. Metagenomics reveals sediment microbial community response to Deepwater Horizon oil spill. ISME J. 2014;8(7):1464-1475.

12. Ayandiran TA, Ayandele AA, Dahunshi SO, et al. Microbial assessment and prevalence of antibiotic resistance in polluted Oluwa River, Nigeria. Egypt J Aquat Res. 2014;40(3):291-299.

13. Yang J, Wang C, Shu C, et al. Marine sediment bacteria harbor antibiotic resistance genes highly similar to those found in human pathogens. Microb Ecol. 2013;65(4):975-981.

14. Tully BJ, Sachdeva R, Graham ED, et al. 290 Metagenome-assembled genomes from the mediterranean sea: ongoing effort to generate genomes from the tara oceans dataset. bioRxiv. 2016.

15. Tully BJ, Heidelberg JF. Potential mechanisms for microbial energy acquisition in oxic deep sea sediments. Appl Environ Microbiol. 2016;82(14):4232-4243. 